\title{
Dynamical Mean-Field Theory for Doped Antiferromagnets
}

\author{
Marcus Fleck \\ Max-Planck-Institut für Festkörperforschung, Heisenbergstrasse 1, D-70569 Stuttgart, Germany \\ Alexander I. Lichtenstein \\ Forschungszentrum Jülich, D-52425 Jülich, Germany \\ Andrzej M. Oles: and Lars Hedin \\ Max-Planck-Institut für Festkörperforschung, Heisenbergstrasse 1, D-70569 Stuttgart, Germany \\ Vladimir I. Anisimov \\ Institute of Metal Physics, Russian Academy of Sciences, 620219 Ekaterinburg, GSP-170, Russia
}

(30 May 1997)

\begin{abstract}
We have generalized the dynamical mean-field theory to study the doping dependence of the crossover from antiferromagnetic to short-range order modelled by an incommensurate spin density wave in the Hubbard model. The local selfenergy which includes spin fluctuations gives quasiparticle weights and spectral properties in good agreement with quantum Monte Carlo and exact diagonalization data in two dimensions. The spectra at finite doping are characterized by a Mott-Hubbard 'gap' accompanied by a pseudogap induced by the local spin order.

PACS numbers: 71.27.+a, 75.10.-b, 74.72.-h, 79.60.-i.
\end{abstract}

Although an important progress in the present understanding of strongly correlated fermion systems occurred recently due to numerical methods, such as quantum Monte Carlo (QMC) and exact diagonalization (ED), an analytic treatment that maintains local correlations is needed to investigate the thermodynamic limit. An attractive possibility is the limit of large spatial dimension $(d=\infty)$, when the diagrams in the perturbative expansion collapse to a single site and the fermion dynamics is described by a local selfenergy [1]. This allows to map lattice models onto quantum impurity models, which can then be solved self-consistently in the dynamical meanfield theory (DMFT) [2]. At large Coulomb interaction $U$, the spectral properties of Mott insulators were reproduced using the DMFT both at half- 2, and at arbitrary filling $n$ [3], but magnetic long-range order (LRO) was analyzed at $d=\infty$ only at half-filling $(n=1)$ [ [ 1 .

The modification of the magnetic order under doping $\delta=1-n$ away from $n=1$ is a central issue in the theory of the oxide superconductors. Undoped $\mathrm{La}_{2} \mathrm{CuO}_{4}$ is a commensurate antiferromagnetic (AF) insulator, while doping by $\mathrm{Sr}$ into $\mathrm{La}_{2-x} \mathrm{Sr}_{x} \mathrm{CuO}_{4}$ results in short-range $\mathrm{AF}$ order within incommensurate magnetic structures [5]. The physical properties of doped $\mathrm{CuO}_{2}$ planes of the high- $T_{c}$ superconductors can be successfully described near $n=1$ using a two-dimensional (2D) single-band Hubbard (or $t-J$ [6]) model, and indeed incommensurate magnetic order was found analytically [7], in Hartree-Fock (HF) [8], and in slave-boson approximation [9.10]. Motivated by the successful description of the transport properties of the $\mathrm{CuO}_{2}$ planes within the DMFT [11,2], we present in this Letter a generalization of the DMFT to the magnetically ordered states, using the Berk-Schrieffer [12] spin-fluctuation exchange interaction with an effective potential due to particle-particle scattering [13], and construct a selfenergy which leads to an overall good agreement with the QMC and ED data. It demonstrates a combination of physics arising from the Slater picture and the Mott-Hubbard description of strongly correlated electron systems.

In order to simulate the weakened short-range AF order in the doped systems, we consider an incommensurate spin spiral (SS) structure. The direction of the magnetization $m$ at site $i$, with respect to the global $z$ axis is specified by two spherical angles, $\Omega_{i}=\left(\phi_{i}, \theta_{i}\right)$. Thus, we transform the original fermions $\left\{a_{i \uparrow}^{\dagger}, a_{i \downarrow}^{\dagger}\right\}$ to the fermions quantized with respect to local quantization axis at each site [10], $c_{i \sigma}^{\dagger}=\sum_{\lambda}\left[\mathcal{R}\left(\Omega_{i}\right)\right]_{\sigma \lambda} a_{i \lambda}^{\dagger}$, where $\mathcal{R}\left(\Omega_{i}\right)=e^{-i\left(\phi_{i} / 2\right) \hat{\sigma}_{z}} e^{-i\left(\theta_{i} / 2\right) \hat{\sigma}_{y}}$, and $\hat{\sigma}_{y}$ and $\hat{\sigma}_{z}$ are Pauli spin matrices. This transforms the Hubbard Hamiltonian with nearest-neighbor hopping, $t_{i j}=-t$, to

$$
H=\sum_{i j, \sigma \sigma^{\prime}} t_{i j} c_{i \sigma}^{\dagger}\left[\mathcal{R}^{*}\left(\Omega_{i}\right) \mathcal{R}\left(\Omega_{j}\right)\right]_{\sigma \sigma^{\prime}} c_{j \sigma^{\prime}}+U \sum_{i} n_{i \uparrow} n_{i \downarrow} .
$$

We take the polar angle as site-independent, $\theta_{i}=\theta$, and let the azimuthal angle describe a spin spiral with a wave-vector $\mathbf{Q}, \phi_{i}=\mathbf{Q} \cdot \mathbf{R}_{i}$. By a straightforward diagonalization of the kinetic energy one finds the energies,

$$
\begin{aligned}
\hat{T}_{\mathbf{Q}}(\mathbf{k}) & =\frac{1}{2} \varepsilon_{\mathbf{k}-\frac{\mathbf{Q}}{2}}\left(\hat{1}+\cos \theta \hat{\sigma}_{z}-\sin \theta \hat{\sigma}_{x}\right) \\
& +\frac{1}{2} \varepsilon_{\mathbf{k}+\frac{\mathbf{Q}}{2}}\left(\hat{1}-\cos \theta \hat{\sigma}_{z}+\sin \theta \hat{\sigma}_{x}\right),
\end{aligned}
$$

where $\varepsilon_{\mathbf{k}}=-2 t\left(\cos k_{x}+\cos k_{y}\right)$. We limit ourselves to plane spirals [14], and choose $\theta=\pi / 2$. 
The one-particle Green function is described by a $(2 \times 2)$ matrix $\hat{G}_{\mathbf{Q}}\left(\mathbf{k}, i \omega_{\nu}\right)$ in spin space, where $\omega_{\nu}$ are fermionic Matsubara frequencies. We approximate the Green function using a local selfenergy [1.2],

$$
\hat{G}_{\mathbf{Q}}^{-1}\left(\mathbf{k}, i \omega_{\nu}\right)=i \omega_{\nu}+\mu-\hat{T}_{\mathbf{Q}}(\mathbf{k})-\hat{\Sigma}_{\mathbf{Q}}^{\mathrm{HF}}-\hat{\Sigma}_{\mathbf{Q}}^{\mathrm{SF}}\left(i \omega_{\nu}\right) .
$$

The local lattice Green function, $\hat{G}_{\mathbf{Q}}\left(i \omega_{\nu}\right)=$ $N_{k}^{-1} \sum_{\mathbf{k}} \hat{G}_{\mathbf{Q}}\left(\mathbf{k}, i \omega_{\nu}\right)$, becomes diagonal due to the parity of $\hat{T}_{\mathbf{Q}}(\mathbf{k})$. Therefore, all local quantities including the selfenergy $\Sigma$, which consists of the HF part, $\Sigma_{\mathbf{Q} \sigma}^{\mathrm{HF}}=U\left\langle n_{0 \bar{\sigma}}\right\rangle$ with $\bar{\sigma}=-\sigma$, and the spin-fluctuation (SF) part, $\Sigma_{\mathbf{Q} \sigma}^{\mathrm{SF}}\left(i \omega_{\nu}\right)$, are diagonal. Using the cavity method [2] for a hypercubic lattice at $d=\infty$, we showed that the dynamical Weiss field, $\mathcal{G}_{\mathbf{Q}, \sigma}^{0}\left(i \omega_{\nu}\right)$, can be computed from the Dyson equation of the spin-symmetry broken Anderson impurity model,

$$
\hat{\mathcal{G}}_{\mathbf{Q}}^{0}\left(i \omega_{\nu}\right)^{-1}=\hat{G}_{\mathbf{Q}}\left(i \omega_{\nu}\right)^{-1}+\hat{\Sigma}_{\mathbf{Q}}^{\mathrm{SF}}\left(i \omega_{\nu}\right) .
$$

The last and the most important step is to find an appropriate expression for selfenergy. This is known to be notoriously difficult and the usual approach within the iterative perturbation scheme (IPS) [3] is to develop an ansatz which reproduces the exact results in certain limits. In spite of the correct large $U$ limit [3], the absence of a reliable form of $\Sigma$ hindered further applications of the DMFT for the magnetic states in the intermediate $U$ range. By considering the Kadanoff-Baym potential containing a class of diagrams up to infinite order [15], we constructed the SF part of selfenergy,

$$
\begin{aligned}
\Sigma_{\mathbf{Q} \sigma}^{\mathrm{SF}}\left(i \omega_{\nu}\right) & =\bar{U}^{2} \beta^{-1} \sum_{\mu} \chi_{\bar{\sigma} \sigma \mathbf{Q}}\left(i \omega_{\mu}\right) \mathcal{G}_{\mathbf{Q} \bar{\sigma}}^{0}\left(i \omega_{\nu}-i \omega_{\mu}\right) \\
& +\bar{U}^{2} \beta^{-1} \sum_{\mu} \chi_{\bar{\sigma} \bar{\sigma}, \mathbf{Q}}\left(i \omega_{\mu}\right) \mathcal{G}_{\mathbf{Q} \sigma}^{0}\left(i \omega_{\nu}-i \omega_{\mu}\right)
\end{aligned}
$$

with $\beta=1 / k_{B} T$. Here we approximated $\Sigma[G]$ by $\Sigma\left[\mathcal{G}^{0}\right]$. Eq. (5) resembles the selfenergy derived by the coupling of the moving hole to transverse spin-fluctuations [16], but we note that the coupling to longitudinal spinfluctuations gives a significant contribution. The selfenergy in a magnetic system is calculated using the symmetry-broken magnetic HF state. The transverse, $\chi_{\bar{\sigma} \sigma}=\bar{U}\left[\chi_{\bar{\sigma} \sigma}^{(0)}\right]^{2} /\left[1-\bar{U} \chi_{\bar{\sigma} \sigma}^{(0)}\right]$, and longitudinal, $\chi_{\sigma \sigma}=$ $\chi_{\sigma \sigma}^{(0)} /\left[1-\bar{U}^{2} \chi_{\uparrow \uparrow}^{(0)} \chi_{\downarrow \downarrow}^{(0)}\right]$, susceptibility in Eq. (5) are found in random phase approximation (RPA) with renormalized interaction $\bar{U}$. Thus, the renormalized interaction $\bar{U}$ is not a fitting parameter [17], but results from the screening by particle-particle diagrams [13,15,

$$
\bar{U}=U /\left[1+U \chi^{p p}(0)\right],
$$

where $\chi^{p p}(0)=\beta^{-1} \sum_{\mu} \mathcal{G}_{\mathbf{Q} \uparrow}^{0}\left(i \omega_{\mu}\right) \mathcal{G}_{\mathbf{Q} \downarrow}^{0}\left(-i \omega_{\mu}\right)$. This screening effect gives the magnetic structure factor 13] and the selfenergy [17] calculated from Eq. (5) in good agreement with the QMC results, and depends on the underlying magnetic order. It is largest in the paramagnetic states and vanishes in the Néel state at $n=1$ for $U \rightarrow \infty$, and is thus very important when the magnetic phase diagrams are considered [15].

Eqs. (3), (4), (5), and (6) represent a solution for the one-particle Green function within the DMFT in the IPS. They have been solved self-consistently and the energetically stable spiral configuration was found. This procedure is further justified by the sum rule [18],

$$
\frac{1}{2 \beta} \sum_{\nu \sigma} \Sigma_{\sigma}\left(i \omega_{\nu}\right) G_{\sigma}\left(i \omega_{\nu}\right) e^{i \omega_{\nu} 0^{+}}=U\left\langle n_{0 \uparrow} n_{0 \downarrow}\right\rangle,
$$

which is well fulfilled in the present approach with $U\left\langle n_{0 \uparrow} n_{0 \downarrow}\right\rangle \simeq \bar{U}\left\langle n_{0 \uparrow}\right\rangle\left\langle n_{0 \downarrow}\right\rangle[15]$. We also show below that the local selfenergy (5) leads to an overall satisfactory agreement with the QMC and ED data.

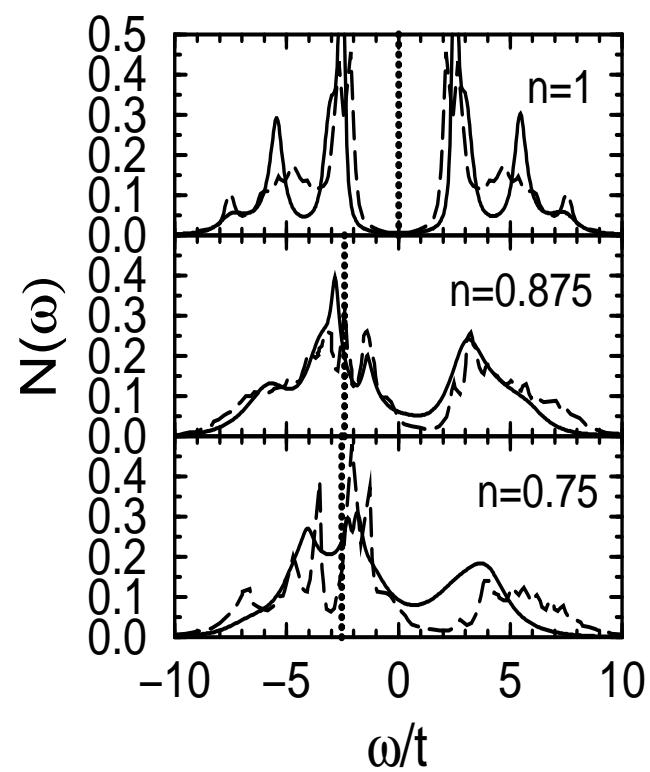

FIG. 1. Total densities of states $N(\omega)$ as obtained within DMFT (solid lines) for $\delta=0$ (AF state), 0.125 [(1,1) spiral], and $0.25[(1,0)$ spiral $]$ with $U / t=8$ and $T=0.05 t$. The dashed lines reproduce $N(\omega)$ found by ED of a $4 \times 4$ lattice in Ref. [22], and the dotted lines show the Fermi energy.

The system with local selfenergy is not fully $2 \mathrm{D}$, and calculations performed at low temperature $T=0.05 t$ $\left(T \ll J=4 t^{2} / U\right)$ converge to states with magnetic LRO, and we discuss here the case of $U / t=8$. Away from halffilling one finds incommensurate states characterized by $\mathbf{Q}=\left[\pi\left(1-2 \eta_{x}\right), \pi\left(1-2 \eta_{y}\right)\right], \eta_{x}=\eta_{y} \neq 0$ for $\operatorname{SS}(1,1)$ and $\eta_{x} \neq 0, \eta_{y}=0$ for $\mathrm{SS}(1,0)$, the neighboring spins are not strictly antiparallel, the kinetic energy $\sim t \sin 2 \pi \eta_{\alpha}$ is gained in agreement with polaron physics, and the dynamics becomes closer to free electrons. We reproduce a typical pattern found in HF and in slave-boson calculations [9,10]: First, the AF state changes to a mixed AF-SS $(1,1)$ state in the range of $0<\delta<0.116$, which indicates that a domain-wall phase [8] might be more stable 
at low doping. Next, a first order transition to a $\mathrm{SS}(1,0)$ is found at $\delta \simeq 0.23$, and both spirals coexist in the range $0.203<\delta<0.248$. The moments decrease under doping, and finally disappear continuosly at $\delta \simeq 0.65$.

Unlike in the IPS based on second order perturbation theory [2], the AF order $[\mathbf{Q}=(\pi, \pi)]$ survives at $n=1$ for $U>4 t$, as found in QMC at $d=\infty$ [20]. In agreement with experiment [21], the spectral functions at $n=1$, $A(\mathbf{k}, \omega)=-\pi^{-1} \sum_{\sigma \sigma^{\prime}} \operatorname{Im} G_{\mathbf{Q}, \sigma \sigma^{\prime}}(\mathbf{k}-\mathbf{Q} / 2, \omega+i \epsilon)$, have the same generic shape as in $t-J$ model [6]19. The photoemission spectrum consists of a coherent quasiparticle $(\mathrm{QP})$ state near the gap with a dispersion $\sim 2 J$, and an incoherent part of width $\sim 7 t$ at lower energies. The DMFT reproduces the nesting instability towards the AF order at $n=1$, but the AF gap is considerably reduced. At $U / t=8$ the HF gap of $7.14 t$ is reduced to $\Delta \simeq 4.93 t$, and agrees very well with the ED data for the $4 \times 4$ cluster [22] (see Fig. 1). In contrast, the gap reduction is overestimated within approaches [16] not treating properly local correlations (7).

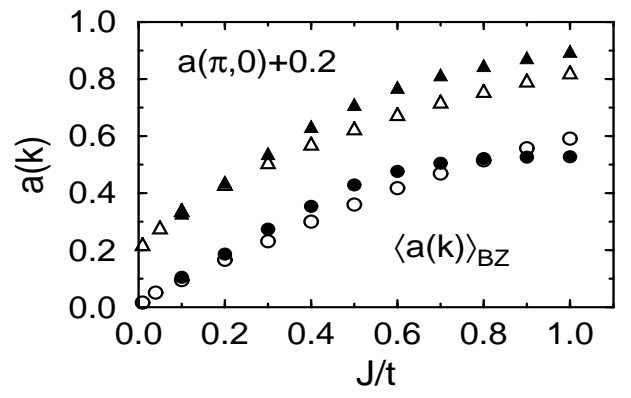

FIG. 2. Spectral weight of a coherent QP at $\mathbf{k}=(\pi, 0)$, $a(\pi, 0)+0.2$ (triangles), and the averaged weight over Brillouin zone, $\langle a(\mathbf{k})\rangle_{\mathrm{Bz}}$ (circles), as obtained at $n=1$ in DMFT (full symbols) and in SCBA (empty symbols) of Ref. [23].

The QP weight $a(\mathbf{k})$ increases with increasing $J / t$, in agreement with the self-consistent Born approximation (SCBA) (and ED data) [23] in the range $J / t<0.5$, both at the $X=(\pi, 0)$ point, and for the averaged weight $\langle a(\mathbf{k})\rangle_{\text {BZ }}$ (Fig. 2). The latter first increases with $J / t$, as in the $t-J$ model, but then flattens out around $J / t \simeq 0.8$, and starts to saturate at a value lower than one. This behavior follows in first place from the absence of the intersite SFs, but also shows that the $t-J$ model does not apply for $J / t>0.8$, where the charge excitations to the upper Hubbard band (UHB) become important.

The quality of our approach is demonstrated by the overall shapes of the densities of states $N(\omega)=$ $N_{k}^{-1} \sum_{\mathbf{k}} A(\mathbf{k}, \omega)$ which are in a very good agreement with the ED data not only at half-filling, but also for doped systems (Fig. 1). At finite doping the system becomes metallic, and the selfenergy (5) exhibits a Fermi-liquid behavior, with the imaginary part $\sim(\omega-\mu)^{2}$ vanishing at the Fermi energy $\mu$. This result might change, however, in a real system due to critical scattering mediated by spin-waves. The spectral weight is transferred from the UHB into the inverse photoemission (IPES) part of the lower Hubbard band (LHB), and $N(\omega)$ agrees very well with the $\mathrm{ED}$ for the $\mathrm{SS}(1,1)$ ground state with $\eta_{x}=\eta_{y} \simeq \delta$ at $\delta=0.125$, both for the positions of the UHB and LHB, and their intensities. We reproduce thereby the increase of the IPES spectral weight above $2 \delta$, being 0.331 at $U / t=8$ [24]. A somewhat smaller distance between the LHB and the UHB for the SS $(1,0)$ at $\delta=0.25$ might suggest that the system properties are closer to a disordered local moment state.
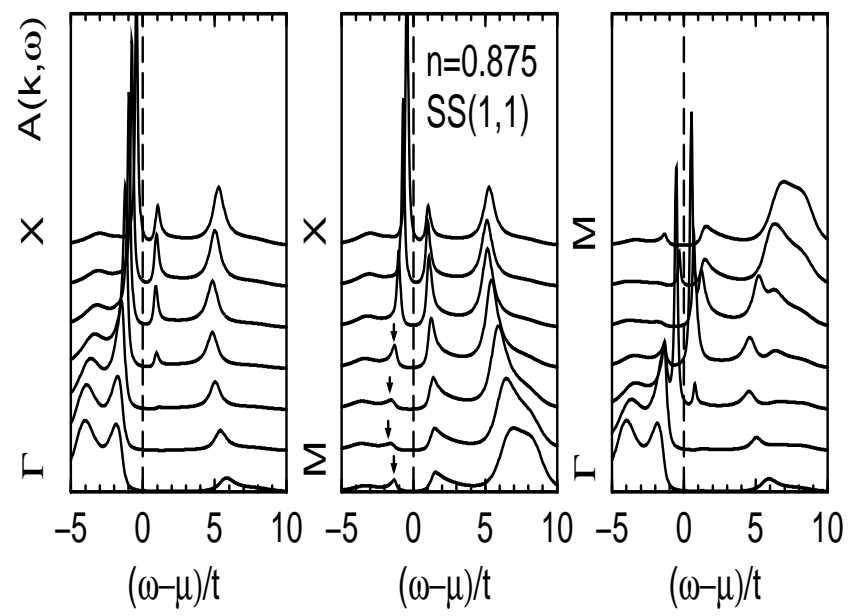

FIG. 3. Spectral functions $A(\mathbf{k}, \omega)$ in the main BZ directions: $\Gamma-X, M-X$, and $\Gamma-M$ in $\mathrm{SS}(1,1)$ state at $\delta=0.125$ and $T=0.05 t$. The spectra along the $\Gamma-M$ direction have been averaged over the $(1,1)$ and $(\overline{1}, \overline{1})$ spirals. A shadow band in $M-X$ direction is indicated by arrows.

The $\mathbf{k}$-resolved spectral functions $A(\mathbf{k}, \omega)$ allow to clarify the generic features of the doped systems: a large Mott-Hubbard gap persists between the LHB and UHB (with energies $\sim-3.3 t, \sim 6.2 t$ in Fig. 3 , respectively), while a new smaller pseudogap forms between the photoemission and IPES parts of the LHB [25]. The latter is induced by the underlying spiral magnetic order which removes the degeneracy between $X$ and $(\pi / 2, \pi / 2)$ points at $\delta=0.125$, and separates two features which originate from a single QP peak at $n=1$; the one with a higher intensity indicates the position of the QP, while the other represents a shadow band. These structures and the pseudogap are best visible in the $X-M$ direction. At the $X$ point one finds the $\mathrm{QP}$ peak at energy $\simeq-0.44 t$ and a pseudogap which persists along the $X-M$ direction, while the spectral weight moves gradually from the lower to the upper maximum 26. Thus, the QP peak at $X$ point tranforms gradually into a shadow band of width $\sim 2 J$ when the $M$ point is approached (see Fig. 3). This interpretation is supported by an excellent agreement with the ED data of Ref. [22]. The QP intensities found at $\delta=0.125$ are somewhat reduced from those in the AF state at $n=1$, and agree qualitatively with ED for the $t-J$ model at the same doping [19]. 
Although we cannot attempt a quantitative comparison within the $t-U$ model, our results agree qualitatively with the $\mathrm{QP}$ band with dispersion $\sim 2 J$ and pseudogap observed in $\mathrm{Bi}_{2} \mathrm{Sr}_{2} \mathrm{CaCu}_{2} \mathrm{O}_{8+\delta} 21$.

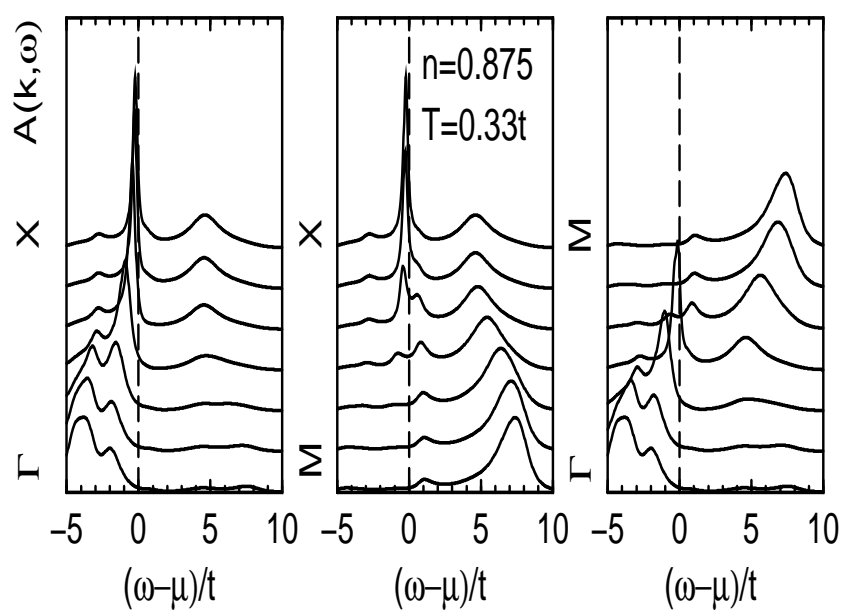

FIG. 4. Spectral functions $A(\mathbf{k}, \omega)$ at $\delta=0.125$ and $T=0.33 t$. The conventions are the same as in Fig. 3 .

The dependence of the QPs on the spiral order explains why the ED results obtained for relatively small clusters [6] cannot reproduce the experimental observations that the QP peak at $X$ point moves above $\omega=\mu$ with increasing doping [27]. Furthermore, the pseudogap gradually disappears with increasing temperature, and the spectral weight crosses the Fermi energy along the $X-M$ direction, as found at $T=0.33 t$ (Fig. (1). This is consistent with the recent QMC calculations [28], suggesting that the pseudogap $\sim J$ originates from short-range magnetic correlations at $T \rightarrow 0$. The spectral functions at $T=0.5 t$ have only broad maxima which correspond to the LHB and UHB, and agree remarkably well with the QMC results of Bulut et al. [29]. At $\delta=0.25$ the spectra consist of incoherent processes even at low $T$, accompanied by new dispersive features $\sim t$, which demonstrates that the pseudogap and the hole-spin correlations are gradually lost in the overdoped regime.

Summarizing, we have developed a simple but very powerful analytic method which captures the essential changes of the electronic structure, and gives coherent QPs by dressing a moving hole with SFs. While the large Mott-Hubbard gap remains almost unchanged with increasing doping, the QP states split into two features separated by a pseudogap. We believe that the electronic properties are generic and not changed by domain-wall formation [8]. It is expected that the present extension of DMFT would significantly improve the understanding of the strongly correlated transition metal oxides, if implemented into realistic band structure calculations.

We thank P. Horsch, G. Kotliar, A. O. Anokhin, and A. I. Poteryaev for valuable discussions, and acknowledge the support by the Committee of Scientific Re- search (KBN) of Poland, Project No. 2 P03B 11813 (AMO), and by the Russian Foundation for Basic Research (RFFI), Grant No. 96-02-16167 (VIA).

* Permanent address: Institute of Physics, Jagellonian University, Reymonta 4, PL-30059 Kraków, Poland.

[1] W. Metzner and D. Vollhardt, Phys. Rev. Lett. 62, 324 (1989); E. Müller-Hartmann, Z. Phys. B 74, 507 (1989).

[2] A. Georges et al., Rev. Mod. Phys. 68, 13 (1996).

[3] H. Kajueter and G. Kotliar, Phys. Rev. Lett. 77, 131 (1996).

[4] D. E. Logan et al., Phys. Rev. Lett. 76, 4785 (1996).

[5] J. M. Tranquada, cond-mat/9702117 P. Dai, H. A. Mook, and F. Doğan, cond-mat/9707112.

[6] E. Dagotto, Rev. Mod. Phys. 66, 763 (1994).

[7] B. I. Shraiman and E. D. Siggia, Phys. Rev. Lett. 62, 1564 (1989); Phys. Rev. B 40, 9162 (1989).

[8] J. Zaanen and O. Gunnarsson, Phys. Rev. B 40, 7391 (1989); H. J. Schulz, Phys. Rev. Lett. 64, 1445 (1990).

[9] C. L. Kane et al., Phys. Rev. B 41, 2653 (1990).

[10] E. Arrigoni and G. C. Strinati, Phys. Rev. B 44, 7455 (1991); R. Frésard and P. Wölfle, J. Phys. Cond. Mat. 4, 3625 (1992).

[11] T. Pruschke et al., Adv. Phys. 44, 187 (1995).

[12] N. F. Berk and J. R. Schrieffer, Phys. Rev. Lett. 17, 433 (1966).

[13] L. Chen et al., Phys. Rev. Lett. 66, 369 (1991).

[14] Double spirals were shown to be unstable in the 2D $t-J$ model in Ref. 9].

[15] M. Fleck et al., Phys. Rev. B 56, 3159 (1997).

[16] J. Altmann et al., Phys. Rev. B 52, 7395 (1995).

[17] N. Bulut et al., Phys. Rev. B 47, 2742 (1993).

[18] Y. M. Vilk and A. M. S. Tremblay, cond-mat/9702188.

[19] W. Stephan and P. Horsch, Phys. Rev. Lett. 66, 2258 (1991).

[20] M. Ulmke et al., Phys. Rev. B 51, 10411 (1995).

[21] B. O. Wells et al., Phys. Rev. Lett. 74, 964 (1995).

[22] E. Dagotto et al., Phys. Rev. B 46, 3183 (1992).

[23] G. Martínez and P. Horsch, Phys. Rev. B 44, 317 (1991).

[24] A similar IPES spectral weight in the LHB increased by kinetic effects to 0.345 is found at $U / t=8$ using an analytic method in one dimension [H. Eskes and A. M. Oleś, Phys. Rev. Lett. 73, 1279 (1994)].

[25] The correct size of the pseudogap induced by spiral order follows from the selfenergy with screened $U(\mathrm{~g})$, while replacing $\bar{U}^{2}$ by $\bar{U} U$ in Eq. (B) [18] destroys spiral ordering and the pseudogap.

[26] The QPs and the weight distribution are expected to be strongly modified in the $t-t^{\prime}-t^{\prime \prime}-J$ model, and thus an extension of the present model might be necessary for a quantitative explanation of recent experiments [R. Eder et al., Phys. Rev. B 55, R3414 (1997)].

[27] D. S. Marshall et al., Phys. Rev. Lett. 76, 4841 (1996).

[28] R. Preuss et al., Phys. Rev. Lett. 79, 1122 (1997).

[29] N. Bulut et al., Phys. Rev. B 50, 7215 (1994). 